\title{
Das Konzept des Kantons Zug für die zweite Welle von COVID-19
}

\author{
Brian Martin ${ }^{a}$, Hanspeter Klaey ${ }^{b}$, Andreas Müller ${ }^{c}$, Christof Gügler $^{d}$, Aurel Koepflie, Beatrice Gross ${ }^{f}$, \\ Martin Pfisterg, Rudolf Hauri ${ }^{\text {h }}$
}

a PD Dr. med., Kantonsarzt adjoint, Amt für Gesundheit Zug, Institut für Epidemiologie, Biostatistik und Prävention der Universität Zürich; b Dr. med. stellvertretender Kantonsarzt, Amt für Gesundheit Zug; ${ }^{c}$ Leiter Rettungsdienst des Kantons Zug; ${ }^{d}$ Beauftragter für gesundheitspolitische Fragen, Gesund-

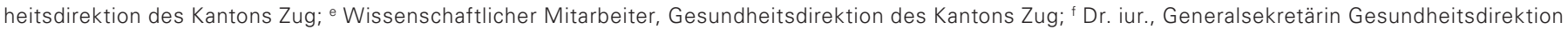
des Kantons Zug; ${ }^{g}$ Regierungsrat und Gesundheitsdirektor des Kantons Zug; ${ }^{h}$ Dr. med., Kantonsarzt, Amtsleiter Amt für Gesundheit Zug, Präsident Vereinigung der Kantonsärztinnen und Kantonsärzte der Schweiz VKS-AMCS

Nachdem die Schweiz die erste Welle der COVID-19-Pandemie im internationalen Vergleich gut überstanden hat, sind für deren weitere Bekämpfung grundsätzlich wieder die Kantone zuständig. Seit Ende Juni 2020 hat der Kanton Zug ein Konzept zur laufenden Beurteilung der Situation, zur Steuerung seiner Massnahmen sowie zur Absprache mit den anderen Kantonen und seinen Partnern auf nationaler Ebene.

Im internationalen Vergleich hat die Schweiz die erste Welle der COVID-19-Pandemie gut überstanden. Das Bundesamt für Gesundheit hat die entsprechenden Erfahrungen in seiner epidemiologischen Zwischenbilanz von Anfang Mai 2020 übersichtlich aufgearbeitet [1]. Die befürchtete Überlastung des Gesundheitswesens ist ausgeblieben, die neu gemeldeten Krankheitsfälle sind seit Anfang April und die mit COVID-19 assoziierten Todesfälle seit Mitte April zurückgegangen.

\section{Das Konzept soll es ermöglichen, eine allenfalls zunehmende Gefährdung der Bevölkerung frühzeitig zu erkennen.}

Die Bekämpfungsmassnahmen hatten einschneidende Wirkungen auf die ganze Gesellschaft, ein grosser Teil der vom Bundesrat erlassenen Einschränkungen sind wieder aufgehoben. Mit der Überführung der ausserordentlichen in die besondere Lage am 16. Juni ist die Verantwortung für die Bekämpfung von COVID-19 grundsätzlich wieder vom Bund an die Kantone übergegangen.

Im Hinblick auf diese Entwicklung hat die Gesundheitsdirektion des Kantons Zug seit Mitte April ein Konzept entwickelt, das es dem Kanton erlauben soll, eine allenfalls wieder zunehmende Gefährdung der Bevölkerung durch COVID-19 frühzeitig zu erkennen, darauf rechtzeitig und angemessen zu reagieren und sich gleichzeitig mit den anderen Kantonen und seinen Partnern auf nationaler Ebene abzusprechen. Die- ses Konzept wurde Ende Juni dem Regierungsrat des Kantons Zug unterbreitet.

Grundsätzlich geht das Konzept davon aus, dass Früherkennung, Contact Tracing und Quarantäne eine wichtige Rolle in der Eindämmung von COVID-19 haben. Gleichzeitig stellt aber aufgrund der nach wie vor bestehenden Verbreitung des Virus und der Möglichkeit von asymptomatischen Erkrankungen eine erneute und auch wieder exponentielle Verbreitung von SARS-CoV-2 nach wie vor eine reale Gefahr dar. Bis ein ursächlich wirksames Medikament oder eine Impfung zur Verfügung stehen, kann diese Gefahr nur durch eine Verringerung der Übertragungsmöglichkeiten in der Bevölkerung gebannt werden, also im Wesentlichen durch das Befolgen der Empfehlungen bezüglich Abstandhalten, Hygiene und Maskentragen.

\section{Alarmstufenkonzept im Kanton Zug}

Das Monitoring der COVID-19-Pandemie umfasst auf nationaler wie auf kantonaler Ebene eine ganze Reihe von Indikatoren, wie sie im Vollzugsmonitoring des Bundesstabs Bevölkerungsschutz im Detail beschrieben werden [2]. Aufgrund der Erfahrungen der ersten Welle sind im Gesundheitswesen die Behandlungskapazitäten limitierend, besonders die Beatmungsplätze auf den Intensivpflegestationen der Spitäler und das entsprechende spezialisierte Pflegepersonal. Die Hospitalisationszahlen zeigen aber in der Regel erst nach 
etwa zwei Wochen die Folgen von vermehrten Ansteckungen, während die Fallzahlen als Grundelement des Monitorings der kantonsärztlichen Dienste wie auch des Bundesamts für Gesundheit im Durchschnitt etwa zwei Tage früher ansteigen [1]. Deswegen basieren die Alarmstufen des Kantons Zug in erster Linie auf diesen Fallzahlen. Als mögliches Frühwarnsystem wer-

\section{Die Alarmstufen des Kantons Zug basieren in erster Linie auf den Fallzahlen.}

den zudem ein automatisches Absenzenmonitoring der Angestellten der Kantonsverwaltung sowie die Messung von Virenbestandteilen im Abwasser geprüft. Die Messwerte für die Alarmstufen des Kantons Zug sind so gewählt, dass sie sowohl auf kantonaler als auch auf überkantonaler Ebene interpretierbar und anwendbar sind. Um oft meldetechnisch bedingte Schwankungen in den Tageszahlen ausgleichen zu können, beruhen die Stufen einerseits auf den kumulierten Fallzahlen der jeweils letzten sieben Tage sowie auf dem Ausmass ihrer Zunahme im Vergleich zu den vorhergehenden sieben Tagen (Tab. 1). Diese prozentuale Zunahme ist ähnlich wie die effektive Reproduktionszahl $\mathrm{R}_{\mathrm{e}}$, die regelmässig von der Swiss National COVID-19 Science Task Force veröffentlicht wird [3], ein Mass für die aktuelle Dynamik der Ausbreitung der Pandemie. Im Gegensatz zu dieser lässt sich aber die prozentuale Zunahme ohne weitere Modellierungen direkt berechnen.
Bei einer Inzidenz von weniger als 10 bestätigten Fällen in den letzten sieben Tagen pro 100000 Einwohner (für den Kanton Zug entsprechend 13 Fällen pro Woche oder durchschnittlich 2 pro Tag, für die Schweiz entsprechend 855 oder 122 Fällen) gilt grundsätzlich Stufe "grün». Diese Zahlengrenze entspricht auch der tiefsten dargestellten Inzidenzstufe von 20 Fällen/ (100000*14 Tage) der Übersichtskarten des European Centre for Disease Prevention and Control ECDC [4]. Bei bis zu 25 Fällen/100 000 wechselt die Stufe auf "gelb», sobald die Fallzahlen innert einer Woche um $10 \% \mathrm{zu}$ genommen haben, sowie auf «orange», sobald sie um mindestens 20\% zugenommen haben. Bei über 25 Fällen/100000 gilt auch unter 10\% Zunahme bereits "gelb», ab 50 Fällen/100000 (in Deutschland entsprechend der Rückfallklausel für den regionalen Notfallplan) «orange» und ab 75 Fällen/100 000 «rot». Die entsprechenden Zahlengrenzen sind gerade angesichts der relativ starken zu erwartenden Schwankungen in einem kleinen Kanton nicht im Sinne eines Automatismus zu verwenden, sondern als Orientierungshilfe zu verstehen.

Eine nachträgliche Anwendung der Alarmstufen auf den Verlauf der ersten Welle für den Kanton Zug und für die gesamte Schweiz findet sich in Abbildung 1. Auch mit der Einschränkung, dass die Testindikatoren und die Testverfügbarkeit sich im Verlauf der Zeit geändert haben, scheint das Alarmstufenkonzept grundsätzlich nachvollziehbar.

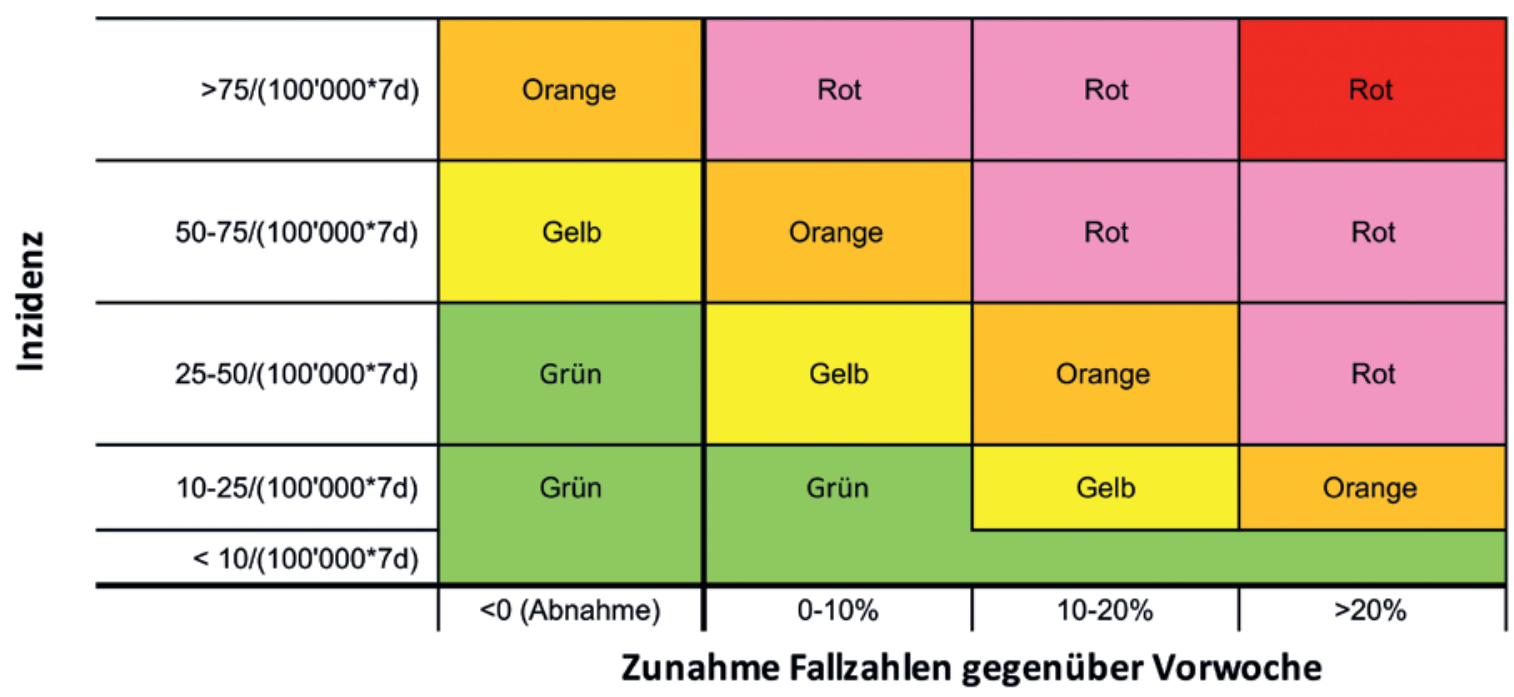

Tabelle 1: Alarmstufen des Konzepts COVID-19 des Kantons Zug. Die Stufen beruhen auf der Verbreitung des Virus in Form der kumulierten Fallzahlen der jeweils letzten 7 Tage sowie auf dem Ausmass der Veränderung im Vergleich zu den vorhergehenden 7 Tagen. Alle Zahlen lassen sich aufgrund öffentlich zugänglicher Daten berechnen. 


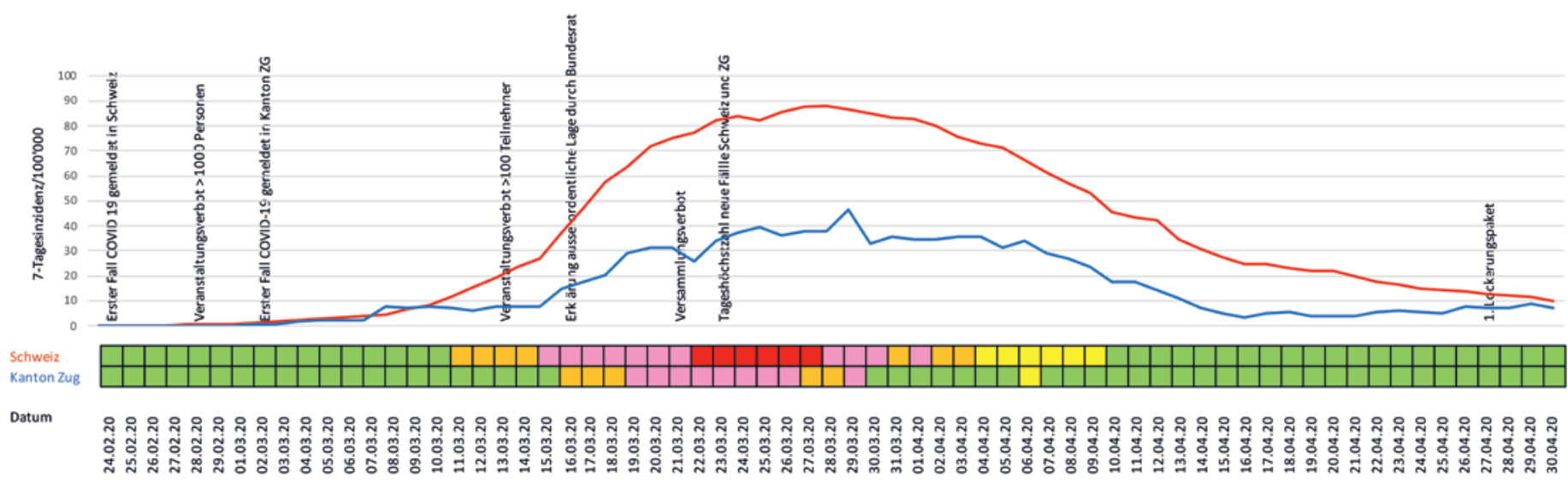

Abbildung 1: Nachträgliche Anwendung der Zuger Alarmstufen auf die Falldaten der ersten Welle der COVID-19-Pandemie in der Schweiz und im Kanton Zug. Im Hinblick auf die Interpretation ist zu berücksichtigen, dass die Testkriterien während der ersten Welle teilweise strenger und die PCR-Tests vorübergehend nur eingeschränkt verfügbar waren. Von Anfang Mai bis Anfang Juli lag die 7-Tages-Inzidenz sowohl in der Schweiz als auch im Kanton Zug immer unter 10/100000, entsprechend der Alarmstufe "grün». Der phasenweise sehr rasche Wechsel zeigt, dass die Vorbereitung von Massnahmen schon deutlich vor dem eigentlichen Stufenwechsel erfolgen muss.

\section{Massnahmen rechtzeitig vorbereiten}

Die nachträgliche Anwendung der Zuger Alarmstufen auf die erste Welle zeigt auch, dass bis zur Vorbereitung spezifischer Massnahmen nicht der Stufenwechsel abgewartet werden kann. Gemeldete Fallzahlen spiegeln nämlich grundsätzlich das Ansteckungsgeschehen knapp zwei Wochen vor diesem Zeitpunkt wider, so dass auch bei unmittelbar ergriffenen und erfolgreichen Massnahmen deren Auswirkung auf Bevölkerungsebene frühestens nach weiteren zwei Wochen zu erwarten ist. Es ist davon auszugehen, dass während dieser Zeit die beobachtete unerwünschte Entwicklung weiterläuft.

Während der ersten Welle der COVID-19-Pandemie kam in der Schweiz und in anderen Ländern eine ganze

\section{Gemeldete Fallzahlen spiegeln das Ansteckungs- geschehen vor zwei Wochen wider.}

Reihe von verschiedenen bevölkerungsbezogenen Massnahmen zur Anwendung. Auch wenn eine vorgängige Quantifikation der Wirksamkeit der verschiedenen Interventionen in einem konkreten Kontext noch nicht möglich ist, lässt sich aus den Erfahrungen doch ableiten, dass eine wirksame Reaktion auf ein erneutes Ansteigen der Übertragungen auch ohne einen erneuten flächendeckenden Lockdown mit seinen gravierenden Auswirkungen auf die gesamte Gesellschaft möglich sein wird.

Der Kanton Zug geht in seinem Konzept nicht davon aus, dass sich im Voraus eine feste Reihenfolge der bei einer allfälligen Verschärfung der Situation zu ergreifenden Interventionen festlegen lässt. Wie Tabelle 2 zeigt, sind aber in den verschiedenen Alarmstufen grundsätzliche Massnahmen auf Ebene des Monitorings, der Kommunikation, weiterer Interventionen sowie der Zusammenarbeit über den Kanton hinaus vorgesehen. Die wichtigsten interventionsbezogenen Handlungsoptionen sind in Tabelle 3 kategorisch zusammengefasst und den Zuständigkeiten innerhalb des Kantons zugeteilt. Nicht explizit darin aufgeführt sind die Massnahmen der Früherkennung, des Contact Tracing und der Quarantäne, die im Kanton Zug bereits während der ersten Welle kontinuierlich sichergestellt werden konnten. Auch diese Massnahmen mit ihren Aufwuchsmöglichkeiten sind aber Inhalt einer laufend angepassten Checkliste aller ausdifferenzierten Handlungsoptionen, die die Gesundheitsdirektion als Arbeitsinstrument für die Lagebeurteilung und für die Vorbereitung konkreter Aktivitäten verwendet.

\section{Die Rolle der Ärzteschaft}

Das leistungsfähige Gesundheitswesen der Schweiz war eine wichtige Voraussetzung dafür, dass die erste Welle der Pandemie in der Schweiz glimpflicher als in vielen anderen Ländern abgelaufen ist. Dies wird im weiteren Verlauf nicht anders sein, und die entsprechenden Kapazitäten von qualifiziertem Personal, Infrastruktur und Material sind zentral auch im Konzept des Kantons Zug. Ärztinnen und Ärzte sind wichtige Vertrauenspersonen für die gesamte Bevölkerung; sie 
haben auch über die Behandlung der Erkrankten hinaus verschiedene wichtige Rollen: bei der frühzeitigen Identifikation und Testung von Erkrankten, bei deren Instruktion zum Verhalten bis zum Vorliegen des Testergebnisses und der Kontaktierung durch die Gesundheitsbehörden, bei Fragen von durch die Situation verunsicherten Menschen, als Heimärztinnen oder Schulärzte. Zur Illustration der ärztlichen Rolle soll das Contact Tracing dienen, in das für COVID-19 sehr grosse Erwartungen gesetzt werden. Die bestehenden

\section{Ärztinnen und Ärzte sind wichtige Vertrauens- personen für die gesamte Bevölkerung.}

Erfahrungen mit Krankheiten wie Tuberkulose, Masern und Meningokokken zeigen, dass eine erfolgreiche Früherkennung und Nachverfolgung von Expositionen und Folgekontakten nicht nur von den zahlenmässigen Ressourcen für diese Aufgaben abhängen. Gerade bei komplexen Sachverhalten ist es oft eine Herausforderung, sehr persönliche oder gar schambehaftete Tatsachen in Erfahrung bringen zu können. Das Vertrauensverhältnis zu Hausärztin oder Hausarzt kann hier eine wichtige Rolle spielen.

Bei allem Vertrauen in Ärzteschaft, Gesundheitswesen, Contact Tracing und Quarantäne geht das Kon- zept des Kantons Zug aber davon aus, dass durchaus wieder Situationen entstehen können, die bevölkerungsbezogene Massnahmen zur Eindämmung von COVID-19 und zur Reduktion der Kontakte verlangen, und dass spezifische Interventionen auch bereits vor einem Wechsel der Alarmstufe sinnvoll sein können. So hat die Gesundheitsdirektion bereits im Juni begonnen, die Auswirkungen der stetig grösser werdenden Kreise mit engen Kontakten im privaten Umfeld sowie die Entwicklung im bekannten Risikosetting «Partyszene» mit ihren Auswirkungen auf Fall- und Quarantänezahlen zu beobachten. Beim erstmaligen Wechsel auf die Alarmstufe «orange» Anfang Juli waren entsprechende Massnahmen schon eingeleitet oder vorbereitet.

Noch ist nicht absehbar, welche anderen Herausforderungen im weiteren Verlauf der COVID-19-Pandemie auf uns zukommen werden. Der Kanton Zug ist zuversichtlich, mit seinem Konzept diesen grundsätzlich begegnen zu können. Er begrüsst, dass die Grundzüge seines Konzepts auch in die Diskussionen bei der Vereinigung der Kantonsärzte der Schweiz VKS und bei der Gesundheitsdirektorenkonferenz GDK eingeflossen sind, und er hofft so einen Beitrag zu einem koordinierten Vorgehen der Kantone geliefert zu haben.

\begin{tabular}{|c|c|c|c|c|c|}
\hline & Massnahmen & $\begin{array}{l}\text { Stufe } \\
\text { Grün }\end{array}$ & $\begin{array}{l}\text { Alarmstufe } \\
\text { Gelb }\end{array}$ & $\begin{array}{l}\text { Alarmstufe } \\
\text { Orange }\end{array}$ & $\begin{array}{c}\text { Alarmstufe } \\
\text { Rot }\end{array}$ \\
\hline & $\begin{array}{l}\text { Zusammenarbeit über Kantone hinaus } \\
\text { (Absprache mit GDK, BAG, and. Partnern) }\end{array}$ & & & & $x$ \\
\hline & $\begin{array}{l}\text { Koordination interkantonal } \\
\text { (Absprache mit Nachbarkantonen) }\end{array}$ & & & $x$ & $x$ \\
\hline & $\begin{array}{l}\text { Austausch interkantonal } \\
\text { (Information Nachbarkantone) }\end{array}$ & & $x$ & $x$ & $x$ \\
\hline \multirow{3}{*}{ 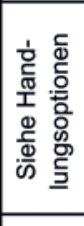 } & Flächendeckende Massnahmen & & & & $x$ \\
\hline & \begin{tabular}{|l} 
Interventionen alle Settings \\
bei spezifischen Hinweisen
\end{tabular} & & & $x$ & $x$ \\
\hline & \begin{tabular}{|l} 
Interventionen Risikosettings \\
bei spezifischen Hinweisen
\end{tabular} & & $x$ & $x$ & $x$ \\
\hline & Kantonale Kommunikation intensivieren & & $x$ & $x$ & $x$ \\
\hline & $\begin{array}{l}\text { Nationale Kommunikation weiterführen } \\
\text { (social distancing/Hygieneregeln) }\end{array}$ & $x$ & $x$ & $x$ & $x$ \\
\hline & Intensivierte Beobachtung alle Settings & & & $x$ & $x$ \\
\hline & Intensivierte Beobachtung Risikosettings & & $x$ & $x$ & $x$ \\
\hline & Pandemieverlaufsmonitoring & $x$ & $x$ & $x$ & $x$ \\
\hline
\end{tabular}

Tabelle 2: Das Konzept sieht in den verschiedenen Alarmstufen grundsätzliche Massnahmen auf Ebene des Monitorings, der Kommunikation, weiterer Interventionen sowie der Zusammenarbeit über den Kanton hinaus vor. Die Handlungsoptionen sind in Tabelle 3 kategorisch aufgelistet. 


\begin{tabular}{|c|c|c|c|}
\hline \begin{tabular}{|l|} 
Handlungsoptionen und Zuständigkeiten im \\
Rahmen Massnahmenkonzept Kanton ZG
\end{tabular} & Kantonsarzt & $\begin{array}{c}\text { Übrige } \\
\text { Verwaltung }\end{array}$ & Gesamtregierungsrat \\
\hline Versammlungsverbote & & & Entscheidung \\
\hline Veranstaltungsverbote & & & Entscheidung \\
\hline Einführung neuer Regelungen (z. B. Maskenpflicht) & & & Entscheidung \\
\hline \multicolumn{4}{|l|}{ Auflagen und Schliessungen } \\
\hline Verkauf und Dienstleistungen & Einzelfall & & über Einzelfall hinaus \\
\hline Restaurants & Einzelfall & & über Einzelfall hinaus \\
\hline Kultur und Sport & Einzelfall & & über Einzelfall hinaus \\
\hline Verkehr und Tourismus & Einzelfall & & über Einzelfall hinaus \\
\hline Bildung & Einzelfall & & über Einzelfall hinaus \\
\hline übrige Wirtschaft & Einzelfall & & über Einzelfall hinaus \\
\hline Betretungsverbote & Gebäude & & über Gebäude hinaus \\
\hline Auflagen oder Schliessungen im Gesundheitswesen & Einzelfall & $\begin{array}{l}\text { Gesundheits- } \\
\text { direktion }\end{array}$ & \\
\hline $\begin{array}{l}\text { Umsetzung bestehender Regelungen } \\
\text { (z. B. Versammlungsverbot) }\end{array}$ & $\begin{array}{l}\text { Inhaltliche } \\
\text { Beratung }\end{array}$ & $\begin{array}{l}\text { zuständige } \\
\text { Behörde }\end{array}$ & \\
\hline $\begin{array}{l}\text { Kommunikation bestehender Empfehlungen } \\
\text { (z. B. Abstandhalten, Hygienemassnahmen) }\end{array}$ & $\begin{array}{l}\text { Inhaltliche } \\
\text { Beratung }\end{array}$ & $\begin{array}{l}\text { Gesundheits- } \\
\text { direktion }\end{array}$ & \\
\hline
\end{tabular}

Tabelle 3: Kategorische Übersicht der wichtigsten interventionsbezogenen Handlungsoptionen im Rahmen des Massnahmenkonzepts COVID-19 des Kantons Zug. Als Arbeitsinstrument besteht eine Checkliste mit einer weiteren Aufgliederung der Optionen, die ihrerseits kontinuierlich im Hinblick auf eine allfällige Umsetzung vorbereitet werden.

Literatur

1 Bundesamt für Gesundheit BAG. Coronavirus-Krankheit 2019 (COVID-19). Epidemiologische Zwischenbilanz zum neuen Coronavirus in der Schweiz und im Fürstentum Liechtenstein. Stand 27.4.2020, 8.00 Uhr. Bern, BAG 2020 (veröffentlicht 7.5.2020).

2 Bundesamt für Bevölkerungsschutz BABS, Bundesstab Bevölkerungsschutz BSTB. Einsatz- \& Supportelement BSTB (NAZ) Vollzugsmonitoring COVID-19. Bericht 3, Lockerungsetappe 3. 19.6.2020, 16.00 Uhr. Bern, BABS 2020.
3 https://ncs-tf.ch/de/lagebericht

4 https://www.ecdc.europa.eu/en/covid-19/country-overviews

\section{Interessenverbindungen}

Alle Interessenverbindungen ergeben sich aus den Affiliationen in der Autorenliste.
PD Dr. med. Brian Martin,

$\mathrm{MPH}$

Facharzt für Prävention und Gesundheitswesen Kantonsarzt adjoint Amt für Gesundheit Aegeristrasse 56 $\mathrm{CH}-6300 \mathrm{Zug}$ brian.martin[at]zg.ch Tel. 0417283939

\section{Das Wichtigste in Kürze}

- Die COVID-19-Alarmstufen im Kanton Zug beruhen auf den kumulierten Fallzahlen der jeweils letzten sieben Tage sowie auf dem Ausmass ihrer Zunahme im Vergleich zu den vorhergehenden sieben Tagen.

- Bei einer Inzidenz von weniger als 10 bestätigten Fällen in den letzten sieben Tagen pro 100000 Einwohner gilt grundsätzlich Stufe "grün»; für den Kanton Zug entspricht dies 13 Fällen pro Woche oder durchschnittlich 2 proTag.

- Die nachträgliche Anwendung der Zuger Alarmstufen auf die erste Welle zeigt, dass bis zur Vorbereitung spezifischer Massnahmen nicht der Stufenwechsel abgewartet werden kann, da die gemeldeten Fallzahlen grundsätzlich das Ansteckungsgeschehen knapp zwei Wochen vor diesem Zeitpunkt widerspiegeln.

- Das Konzept des Kantons Zug geht davon aus, dass durchaus wieder Situationen entstehen können, die bevölkerungsbezogene Massnahmen zur Eindämmung von COVID-19 und zur Reduktion der Kontakte verlangen und dass spezifische Interventionen auch bereits vor einem Wechsel der Alarmstufe sinnvoll sein können.

\section{L'essentiel en bref}

- Dans le canton de Zoug, les niveaux d'alerte COVID-19 reposent sur le nombre cumulé de cas des sept derniers jours et l'ampleur de leur augmentation par rapport aux sept jours précédents.

- Si l'incidence est inférieure à 10 cas confirmés pour 100000 habitants au cours des sept derniers jours, le niveau est en principe "vert". Dans le canton de Zoug, cela correspond à 13 cas par semaine ou 2 cas par jour en moyenne.

- L'application rétrospective des niveaux d'alerte de Zoug à la première vague montre qu'il ne faut pas attendre le changement de niveau avant de préparer des mesures spécifiques, car le nombre de cas signalés reflète généralement la survenue de la contamination, un peu moins de deux semaines avant ce moment.

- Le concept du canton de Zoug part du principe que des situations qui requièrent des mesures impliquant la population en vue d'endiguer la COVID-19 et de réduire les contacts pourraient tout à fait se produire à nouveau et que des interventions spécifiques peuvent s'avérer utiles même avant un changement de niveau d'alerte. 